OPEN ACCESS

Edited by:

Damiano Caputo,

Campus Bio-Medico University, Italy

Reviewed by:

Paolo Gallo,

Policlinico Universitario Campus

Bio-Medico, Italy

Mikel Prieto,

Cruces University Hospital, Spain

*Correspondence:

Wan Yee Lau

josephlau@cuhk.edu.hk

Tao Peng

pengtaogmu@163.com

tThese authors have contributed equally to this work and share first

authorship

Specialty section: This article was submitted to

Surgical Oncology,

a section of the journal

Frontiers in Surgery

Received: 13 May 2021

Accepted: 07 July 2021

Published: 17 September 2021

Citation:

Yu T, YeX, Wen Z, Zhu G, Su H,

Han C, Huang K, Qin W, Liao X,

Yang $C$, Liu Z, Wang $X$, Xu B, Su M,

Lv Z, Lau WY and Peng T (2021)

Intraoperative Indocyanine Green

Retention Test of Left Hemiliver in

Decision-Making for Patients With

Hepatocellular Carcinoma Undergoing

Right Hepatectomy.

Front. Surg. 8:709017.

doi: 10.3389/fsurg.2021.709017

\section{Intraoperative Indocyanine Green Retention Test of Left Hemiliver in Decision-Making for Patients With Hepatocellular Carcinoma Undergoing Right Hepatectomy}

\author{
Tingdong $\mathrm{Yu}^{1,2 t}$, Xinping $\mathrm{Ye}^{1+}$, Zhang Wen ${ }^{1}$, Guangzhi Zhu ${ }^{1}$, Hao Su ${ }^{1}$, Chuangye Han ${ }^{1}$, \\ Ketuan Huang ${ }^{1}$, Wei Qin ${ }^{1}$, Xiwen Liao ${ }^{1}$, Chengkun Yang ${ }^{1}$, Zhen Liu ${ }^{1}$, Xiangkun Wang ${ }^{1}$, \\ Banghao $\mathrm{Xu}^{1}$, Ming Su ${ }^{1}$, Zili $\mathrm{Lv}^{3}$, Wan Yee $\mathrm{Lau}^{4 *}$ and Tao Peng ${ }^{1 *}$ \\ ${ }^{1}$ Department of Hepatobiliary Surgery, the First Affiliated Hospital of Guangxi Medical University, Nanning, China, \\ ${ }^{2}$ Department of Hepatobiliary Surgery, the Third Affiliated Hospital of Kunming Medical University, Kunming, China, \\ ${ }^{3}$ Department of Pathology, the First Affiliated Hospital of Guangxi Medical University, Nanning, China, ${ }^{4}$ Faculty of Medicine, \\ The Chinese University of Hong Kong, Hong Kong, China
}

Introduction: The aim of this study was to select qualified patients with hepatocellular carcinoma $(\mathrm{HCC})$ who underwent right hepatectomy $(\mathrm{RH})$ via intraoperative indocyanine green retention test at 15 min (ICG-R15) of the left hemiliver, which prevents severe posthepatectomy liver failure (PHLF).

Methods: Twenty HCC patients who were preoperatively planned to undergo $\mathrm{RH}$ were enrolled. Intraoperative ICG-R15 of left hemiliver was measured after the right Glissonean pedicle was completely blocked. Patients then underwent RH if intraoperative ICG-R15 was $\leq 10 \%$. Otherwise, patients underwent staged $\mathrm{RH}(\mathrm{SRH})$, either associating liver partitioning and portal vein ligation for staged hepatectomy (ALPPS) or portal vein ligation (PVL), followed by stage-2 $\mathrm{RH}$. The comparison group consisted of patients with a ratio of standard left liver volume (SLLV) of $>40 \%$ and preoperative ICG-R15 $\leq 10 \%$ who underwent $\mathrm{RH}$. The clinical outcomes of these two groups were compared.

Results: Of the 20 patients, six underwent stage- $1 \mathrm{RH}$, six underwent ALPPS, five underwent $\mathrm{PVL}$ followed by stage- $2 \mathrm{RH}$, and three failed to proceed to stage-2 $\mathrm{RH}$ after PVL. No significant differences were found among the 17 patients who underwent stage- 1 or stage-2 $\mathrm{RH}$ in the study group, the 19 patients in the comparison group, the 11 patients in the stage-2 $\mathrm{RH}$ group, and the six patients in the stage- $1 \mathrm{RH}$ group in incidences of PHLF, postoperative complications, hospital stay, and HCC recurrence within 1 year after $\mathrm{RH}$. Compared with the stage-1 ALPPS group, the mean operative time and blood loss of the stage-1 PVL group were significantly less $(p<0.001$ and $p=$ 0.022 , respectively). The stage-1 PVL group had a significantly longer waiting-time (43.4 vs. 14.0 days, $p=0.016$ ) than the stage- 1 ALPPS group to proceed to stage-2 $\mathrm{RH}$. After stage-2 $\mathrm{RH}$, tumor recurrence within 1 year was 20\% (1/5) in patients after PVL and $50 \%(3 / 6)$ after stage-1 ALPPS. 
Conclusions: Intraoperative ICG-R15 $\leq 10 \%$ of left hemiliver was valuable in intraoperative decision-making for patients who were planned to undergo $\mathrm{RH}$. There is a possibility that stage-1 PVL might help to select patients with more favorable biological behavior to undergo stage-2 $\mathrm{RH}$.

Keywords: hepatocellular carcinoma, indocyanine green retention test, hepatectomy, ALPPS, portal vein ligation, future liver remnant, liver function

\section{INTRODUCTION}

Hepatic resection is still the first line treatment for patients with hepatocellular carcinoma (HCC) aiming at cure (1). However, posthepatectomy liver failure (PHLF) and its associated complications remain common after major hepatectomy (2). Right hepatectomy $(\mathrm{RH})$ is a commonly-used surgical procedure for patients with HCC in the right hemiliver. PHLF remains the main cause of hepatectomy-related mortality after RH, especially in patients with a cirrhotic liver (3-5). To accomplish RH for an originally unresectable tumor because of inadequate volume of left hemiliver and to decrease PHLF, portal vein embolization (PVE), portal vein ligation (PVL), and associating liver partitioning and portal vein ligation for staged hepatectomy (ALPPS) are possible surgical strategies to induce remnant left hemiliver hypertrophy (6-8).

The occurrence of PHLF is closely-related to the volume and function of the future remnant left hemiliver (4). Threedimensional (3D) modeling of the liver based on computed tomography (CT) images can provide accurate remnant liver volume measurement $(9,10)$. Indocyanine green retention test at $15 \mathrm{~min}$ (ICG-R15) is a commonly used and a noninvasive assessment of liver function, which has good predictive values for PHLF (11). Indeed, preoperative ICG-R15 tests have been used to select patients for major hepatectomy $(12,13)$. While preoperative 3D measurement calculates the only remnant liver volume that is planned to be left behind after liver resection, preoperative ICG-R15 test measures all the functioning nontumorous parts of the liver, including parts of the liver that are planned to be resected. This study aimed to determine using intraoperative ICG-R15 retention tests to assess only the left hemiliver function in patients who were planned to undergo RH by blocking the right hepatic blood flow. Based on the results of intraoperative ICG-R15 tests, either a stage-1 or stage-2 RH was carried out.

\section{PATIENTS AND METHODS}

\section{Patients}

Between 2015 and 2018, consecutive patients with hepatitis B virus (HBV)-related HCC in the right hemiliver, who were considered for RH at The First Affiliated Hospital of Guangxi Medical University (Guangxi, China), entered into this study. These patients routinely underwent 64-slice spiral contrastenhanced CT scanning. Three dimensional (3D) images of the liver and tumor were then reconstructed, and the standard left liver volume (SLLV) was calculated. Informed consent was obtained for these patients to undergo an intraoperative ICGR15 retention test after blocking the right hepatic blood inflow to select the surgical procedure: either stage-1 or stage- $2 \mathrm{RH}$. When the test was $>10 \%$, the choice of a stage- $2 \mathrm{RH}$ using either stage-1 ALPPS or PVL was determined preoperatively by the patients after discussion with the surgical group. A comparison group of patients who underwent RH during the study period was collected from the hospital database. All these patients had a $>40 \%$ ratio of SLLV and preoperative ICG-R15 $\leq 10 \%$, but no intraoperative indocyanine green (ICG) retention tests. Based on the Chinese Guidelines (14), Child-Pugh grade A and ICGR $15<20-30 \%$ are generally considered as the prerequisites for any hepatectomy. A future remnant liver (FRL) volume of more than $40 \%$ (for patients with a liver background of chronic hepatitis/cirrhosis) or more than $30 \%$ (for patients without that liver background) of the standard remnant liver volume is the prerequisite for major liver resection. Therefore $\mathrm{RH}$ is routinely carried out in our unit for HBV-related HCC patients with $>40 \%$ ratio of SLLV and a preoperative ICG-R15 $\leq 10 \%$.

The clinicopathologic and perioperative data were collected from the hospital database and pathologic reports. All patients had HBV infection. They all had received antihepatitis viral treatment before and after operations. The tumors were staged according to the Barcelona Clinic Liver Cancer (BCLC) staging system (15). Liver fibrosis and necroinflammatory scores of the non-tumorous tissues were evaluated postoperatively using the Ishak scoring system (16). PHLF was defined according to the International Study Group of Liver Surgery on or after postoperative day 5 using the increased international normalized ratio and hyperbilirubinemia, as defined by the normal cut-off levels of the local laboratory (17). Postoperative complications were evaluated using the Clavien-Dindo classification (18). This study was conducted with approval from the Ethics Committee of the First Affiliated Hospital of Guangxi Medical University. Patients gave informed consent for the operations and for their data to be used for clinical research.

\section{Analysis of 3D Reconstruction and Left Liver Volume Measurements}

Computed tomography images, including non-enhanced and three-phase contrast-enhanced scans (arterial, portal venous, and delayed phases), were obtained from a 64-slice spiral CT scanner. The CT scanning data was inputted in IQQA-Liver (EDDA Technology, Princeton, NJ, USA). The spatial structures including the tumor, hepatic artery, portal vein, and liver, were then reconstructed as a 3D model and the actual total liver volume (TLV) and left liver volume (LLV) were automatically 
TABLE 1 | Demography of HCC Patients who underwent RH.

\begin{tabular}{|c|c|c|c|}
\hline Variables & $\begin{array}{l}\text { Intraoperative } \\
\text { ICG-R15 test group } \\
(n=20)\end{array}$ & $\begin{array}{l}\text { Comparison group } \\
(n=19)\end{array}$ & $P$-value \\
\hline Age, yrs & $43.7(35-50)$ & $42.5(20-69)$ & 0.718 \\
\hline Female/male & $2 / 18$ & $5 / 14$ & 0.235 \\
\hline Child-Pugh class A/B & $19 / 1$ & $18 / 1$ & 1.000 \\
\hline \multicolumn{4}{|l|}{ Tumor } \\
\hline $\mathrm{HCC}$ & 19 & 19 & 1.000 \\
\hline HCC-ICC & 1 & 0 & \\
\hline SLLV, ml & $445.8(262.1-613.4)$ & 558.9(343.6-786.2) & 0.002 \\
\hline Ratio of SLLV, \% & $44.3(23.0-66.6)$ & $55.0(41.4-73.8)$ & 0.001 \\
\hline Preoperative ICG-R15, 9 & $67.0(2.1-11.5)$ & $5.3(0.9-9.8)$ & 0.045 \\
\hline \multicolumn{4}{|l|}{ Tumor number, $n$} \\
\hline 1 & 14 & 13 & 1.000 \\
\hline$\geq 2$ & 6 & 6 & \\
\hline Tumor size, cm & $9.2(3.5-17)$ & $11.0(3.0-17)$ & 0.176 \\
\hline \multicolumn{4}{|l|}{ PVTT, $n$ (\%) } \\
\hline । & $2(10.0 \%)$ & $2(10.5 \%)$ & 1.000 \\
\hline$\|$ & $6(30.0 \%)$ & 0 & NA \\
\hline III & 0 & $1(5.3 \%)$ & NA \\
\hline $\mathrm{MVI}$ & $10(62.5 \%)$ & $11(57.9 \%)$ & 1.00 \\
\hline Missing & 3 & 0 & \\
\hline BCLC stage $A / B$ & $15 / 5$ & $12 / 7$ & 0.501 \\
\hline
\end{tabular}

ICG, indocyanine green; HCC, hepatocellular carcinoma; HCC-ICC, combined hepatocellular carcinoma and intrahepatic cholangiocarcinoma; SLLV, standard left liver volume; PVTT, portal vein tumor thrombus; MVI, microvascular invasion; RH, right hepatectomy.

calculated. The SLLV was calculated according to the following formula to estimate the liver volumes of the Chinese patients: standard liver volume $\left(\mathrm{SLV}\left[\mathrm{cm}^{3}\right]\right)=11.508 \times$ body weight $(\mathrm{kg})$ +334.024 and SLLV $=$ SLV $\times$ LLV/TLV (19).

\section{Intraoperative ICG Retention Test and Selection of Surgical Procedures}

Indocyanine green retention test at $15 \mathrm{~min}$ was measured using a nasal mucosa probe of Hitachi 7700 (Tokyo, Japan) by injecting a dose of $0.5 \mathrm{mg} / \mathrm{kg}$ of ICG rapidly via a peripheral vein of the forearm when the right Glissonean pedicle at the hepatic hilus was completely blocked for $5 \mathrm{~min}$ intraoperatively. An ICG-R15 $\leq 10 \%$ of the left remnant liver was considered adequate to proceed to a stage- $1 \mathrm{RH}$. In contrast, patients were planned to undergo a stage- $2 \mathrm{RH}$ when the ICG-R15 was $>10 \%$. The stage1 operations included ALPPS or PVL following the wish of the patients before surgery.

\section{Statistical Analysis}

Quantitative data were expressed as mean and standard deviation and compared using the Student's $t$-test. Qualitative variables are expressed as numbers and percentages and compared using Fisher's exact tests. Spearman's rank-order correlation was used to evaluate the relationship with PHLF, the SLLV ratio, the necroinflammatory scores, and the fibrosis scores. Statistical
TABLE 2 | Outcomes of $\mathrm{RH}$ in intraoperative ICG test group and comparison group.

\begin{tabular}{|c|c|c|c|}
\hline Variables & $\begin{array}{l}\text { Intraoperative } \\
\text { ICG-R15 } \\
\text { test group } \\
(n=20)\end{array}$ & $\begin{array}{l}\text { Comparison } \\
\text { group } \\
(n=19)\end{array}$ & $P$-value \\
\hline \multicolumn{4}{|l|}{ Surgical procedure, $n(\%)$} \\
\hline $\mathrm{RH}$ & 6(30.0\%) & 19(100\%) & $<0.001$ \\
\hline Staged procedure & $14(70.0 \%)$ & 0 & \\
\hline \multicolumn{4}{|l|}{ RH completion, $n$ (\%) } \\
\hline $\mathrm{RH}$ & 6(35.3\%) & 19(100\%) & $<0.001$ \\
\hline $\mathrm{SRH}$ & $11(64.7 \%)$ & 0 & \\
\hline Failure for SRH & 3 & & \\
\hline \multicolumn{4}{|l|}{ Ishak score } \\
\hline Fibrosis stage, $n(\%)$ & & & 0.181 \\
\hline Stage $1+$ stage 2 & $6(35.3 \%)$ & $12(63.2 \%)$ & \\
\hline Stage $3+$ stage 4 & $11(64.7 \%)$ & $7(36.8 \%)$ & \\
\hline Necroinflammatory grade, $n$ (\%) & & & 0.981 \\
\hline Grade 1 & $4(23.5 \%)$ & $4(21.0 \%)$ & \\
\hline Grade 2 & $8(47.1 \%)$ & $9(47.4 \%)$ & \\
\hline Grade 3 & $5(29.4 \%)$ & $6(31.6 \%)$ & \\
\hline $\operatorname{PHLF},(n, \%)$ & & & 0.505 \\
\hline None & $10(58.8 \%)$ & $8(42.1 \%)$ & \\
\hline Grade A+B & $7(41.2 \%)$ & $11(67.9 \%)$ & \\
\hline Clavien-Dindo, $n$ (\%) & & & 0.281 \\
\hline Grade 1 & 10(58.8\%) & $15(78.9 \%)$ & \\
\hline Grade 2 & $7(41.2 \%)$ & $4(21.1 \%)$ & \\
\hline Hospital stay after surgery, days & $14(7-47)$ & 10(6-22) & 0.122 \\
\hline Recurrence within 1 year, $n(\%)$ & $6(35.3 \%)$ & $7(36.8 \%)$ & 1.000 \\
\hline missing & 0 & 1 & \\
\hline
\end{tabular}

$R H$, right hepatectomy; SRH, staged right hepatectomy; PHLF, posthepatectomy liver failure.

analysis was performed using SPSS (version 21.0, IBM), and a $P$-value $<0.05$ was considered statistically significant.

\section{RESULTS}

\section{Patient}

As shown in Table 1, 20 patients with HBV-related HCC in the right hemiliver who required $\mathrm{RH}$ were included into this study. These patients accepted intraoperative ICG retention tests to select between the stage- 1 or stage- 2 RH. Specifically, six patients underwent stage-1 $\mathrm{RH}$ and 14 patients underwent stage-2 $\mathrm{RH}$. During the same time period, 19 patients who had preoperative ICG-R15 $\leq 10 \%$ and adequately determined over $40 \%$ ratio of SLLV underwent stage-1 RH without intraoperative ICG-R15 tests. These patients were used as the comparison group. Our data showed that SLLV $(445.8 \mathrm{~mL})$ and SLLV ratio of $44.3 \%$ in the ICG test group was significantly less compared with the SLLV $(558.9 \mathrm{~mL})$ and SLLV ratio $(55.0 \%)$ in the comparison group ( $p$ $<0.002$ and $p<0.001$, respectively), and the preoperative ICGR15[7.0(2.1-11.5)\%] in ICG test group was higher compared with ICG-R15 [5.3(0.9-9.8)\%] in the comparison group ( $p=$ 


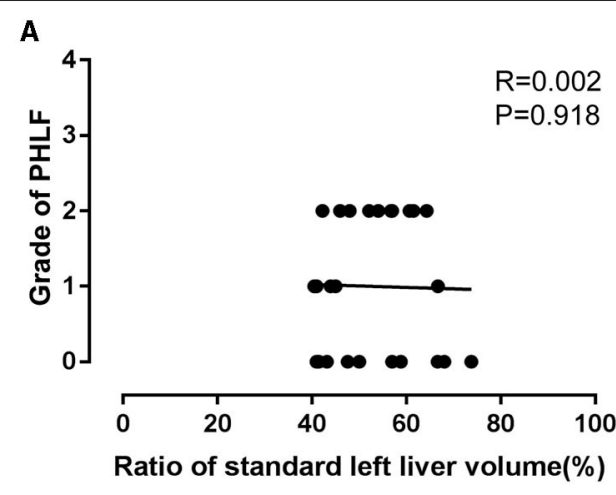

c

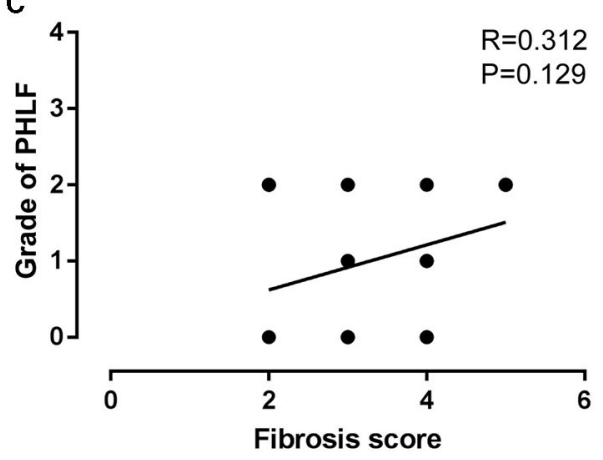

B

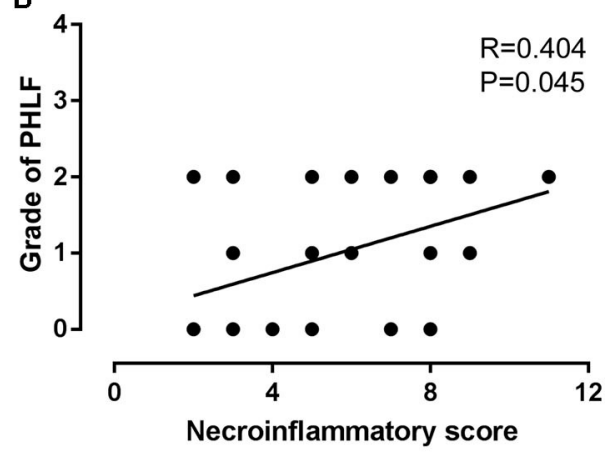

D

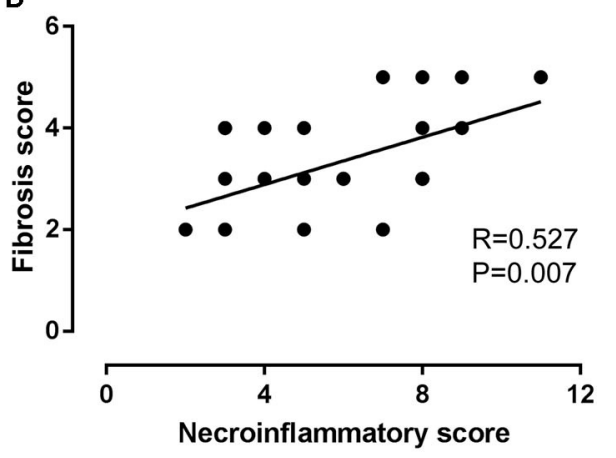

FIGURE 1 | Association analysis of grade of PHLF with ratio of standard left liver volume (A), liver fibrosis score (B) and necroinflammatory score (C), fibrosis score with necroinflammatory score (D) for total 25 patients with $\mathrm{RH}$.

0.045). There were no significant differences between the two groups in age, gender, Child-Pugh grading, tumor number, tumor size, and BCLC staging.

\section{Outcomes of the Intraoperative ICG Retention Test Group and the Comparison Group}

Based on the results of intraoperative ICG-R15 tests, of the 20 patients in the study group (Table 2), six patients finally underwent stage-1 $\mathrm{RH}$ and 14 patients were planned to undergo stage-2 RH using either stage-1 ALPPS $(n=6)$ or stage-1 PVL ( $n$ $=8$ ). Of these 14 patients who were under stage- 1 operations, 11 were able to proceed to stage-2 RH (after stage-1 ALPPS, $n=6$, after stage- 1 PVL, $n=5$ ) because of adequate growth in volumes of the FRL, and the remaining three cases failed for stage-2 RH due to tumor progression. The fibrosis stages $(\mathrm{S} 3+\mathrm{S} 4)$ of the livers in the 17 patients with planned stage$2 \mathrm{RH}(64.7 \%, 11 / 17)$ were more severe, though insignificant, than the 19 patients in the comparison group $(36.8 \%, 7 / 19)$ $(p>0.005)$. However, our results showed that the incidences of PHLF, Clavien-Dindo grading, hospital stay, and recurrence within 1 year after $\mathrm{RH}$ in the intraoperative ICG test group were not significantly worse than the comparison group. A combined analysis of all 25 patients who underwent stage- $1 \mathrm{RH}$ (six in the intraoperative ICG-R15 group and 19 in the comparison group) suggested that PHLF following $\mathrm{RH}$ was significantly associated with the liver necroinflammatory score $(r=0.404, p=0.045$;
Figure 1B), but not with SLLV (Figure 1A) and fibrosis score (Figure 1C). However, the liver fibrosis score was closely related to the liver necroinflammatory score $(r=0.527, p=0.007$; Figure 1D).

\section{Analysis of Stage-2 RH With Intraoperative ICG Retention Tests}

Comparing the 11 patients who underwent stage-2 $\mathrm{RH}$ with the six patients who underwent stage-1 RH based on the intraoperative ICG-R15 tests, no significant differences were found in SLLV and in SLLV ratio. However, the preoperative ICG-R15 and intraoperative ICG-R15 in patients who underwent stage-2 RH were significantly higher than those patients who underwent stage- $1 \mathrm{RH}$ ( $p=0.01$ and $p<0.001$, respectively, Table 3). After the left hemilivers had increased to adequate volumes in the 11 patients who underwent stage-2 $\mathrm{RH}$, the surgical time, blood loss, PHLF grading, postoperative complications, and hospital stay were similar to the six patients who underwent stage- $1 \mathrm{RH}$, with the incidence of PHLF in the stage-2 RH group $(27.3 \%, 3 / 11)$ being less than the stage- $1 \mathrm{RH}$ group $(66.7 \%, 4 / 6)$.

\section{Comparison of Stage-1 Surgery Between the ALPPS and PVL Groups}

The data of the six patients who underwent stage-1 ALPPS and the eight patients who underwent stage-1 PVL are shown in Table 4. In the PVL group, five patients underwent laparoscopic stage-1 operation. The average operative time and blood loss 
TABLE 3 | Outcomes of SRH and $\mathrm{RH}$ with intraoperative ICG-R15 test in the study group.

\begin{tabular}{|c|c|c|c|}
\hline Variables & $\operatorname{SRH}(n=11)$ & RH $(n=6)$ & $P$-value \\
\hline Intraoperative ICG-R15, \% & $\begin{array}{l}14.8 \\
(10.4-24.1)\end{array}$ & $\begin{array}{l}6.6 \\
(2.6-9.2)\end{array}$ & $<0.001$ \\
\hline Preoperative ICG-R15, \% & $\begin{array}{l}7.4 \\
(3.7-11.5)\end{array}$ & $\begin{array}{l}4.1 \\
(2.1-6.3)\end{array}$ & 0.01 \\
\hline SLLV, ml & $\begin{array}{l}428.6 \\
(262.1-568.1)\end{array}$ & $\begin{array}{l}462.6 \\
(349.3-613.4)\end{array}$ & 0.538 \\
\hline SLLV ratio, \% & $\begin{array}{l}42.3 \\
(23.0-52.5)\end{array}$ & $\begin{array}{l}47.2 \\
(41.0-66.6)\end{array}$ & 0.292 \\
\hline SLLV before $\mathrm{RH}, \mathrm{ml}$ & $\begin{array}{l}573.2 \\
(450.1-758.2)\end{array}$ & $\begin{array}{l}462.6 \\
(349.3-613.4)\end{array}$ & 0.027 \\
\hline SLLV ratio before $\mathrm{RH}, \%$ & $\begin{array}{l}56.2 \\
(40.0-67.9)\end{array}$ & $\begin{array}{l}47.2 \\
(41.0-66.6)\end{array}$ & 0.071 \\
\hline Operation time, minutes & $\begin{array}{l}300.7 \\
(213-515)\end{array}$ & $\begin{array}{l}372.5 \\
(217-448)\end{array}$ & 0.234 \\
\hline Blood loss, ml & $\begin{array}{l}981.8 \\
(300-2,500)\end{array}$ & $\begin{array}{l}908 \\
(350-1,600)\end{array}$ & 0.815 \\
\hline \multicolumn{4}{|l|}{ Ishak score } \\
\hline Fibrosis stage, $n(\%)$ & & & 0.600 \\
\hline Stage $1+$ stage 2 & $3(27.3 \%)$ & $3(50.0 \%)$ & \\
\hline Stage $3+$ stage 4 & $8(72.7 \%)$ & $3(50.0 \%)$ & \\
\hline Necroinflammatory grade, $n(\%)$ & & & 0.090 \\
\hline Grade 1 & $1(9.1 \%)$ & $3(50.0 \%)$ & \\
\hline Grade 2 & $7(63.6 \%)$ & $1(16.7 \%)$ & \\
\hline Grade 3 & $3(27.3 \%)$ & $2(33.3 \%)$ & \\
\hline PHLF, $n(\%)$ & & & 0.162 \\
\hline None & $8(72.7 \%)$ & $2(33.3 \%)$ & \\
\hline Grade $A+B$ & $3(27.3 \%)$ & $4(66.7 \%)$ & \\
\hline Clavien-Dindo, $n(\%)$ & & & 1.000 \\
\hline Grade 1 & $6(54.5 \%)$ & $4(66.7 \%)$ & \\
\hline Grade 2 & $5(45.5 \%)$ & $2(33.3 \%)$ & \\
\hline Hospital stay after surgery, days & $15(8-47)$ & $12(7-27)$ & 0.598 \\
\hline Recurrence within 1 year, $n(\%)$ & $3(27.3 \%)$ & $3(50.0 \%)$ & 0.600 \\
\hline missing & 0 & 0 & \\
\hline
\end{tabular}

$R H$, right hepatectomy; $S R H$, staged right hepatectomy; PHLF, posthepatectomy liver failure.

in the PVL group were significantly less compared with the stage-1 ALPPS group ( $p<0.001$ and $p=0.022$, respectively). Two patients developed PHLF and one had bile leakage after the stage-1 procedure in the ALPPS group, whereas three patients in the PVL group failed to proceed to the stage- $2 \mathrm{RH}$ due to tumor progression and inadequate growth in volumes of FRL.

\section{Comparison of Stage-2 RH Between the ALPPS and PVL Groups}

All six patients in the stage-1 ALPPS group and five in the stage1 PVL group completed stage-2 RH. Two patients underwent transarterial chemoembolization (TACE) after the stage-1 PVL because of a long wait for liver hypertrophy to reach adequate volumes in the FRL. Our results demonstrated that patients in the PVL group had to wait for a significantly longer time
TABLE 4 | Comparison of staged-1 clinical characteristics of SRH between the ALPPS group and PVL group.

\begin{tabular}{llll}
\hline Variables & $\begin{array}{l}\text { ALPPS } \\
(\mathbf{N}=6)\end{array}$ & $\begin{array}{l}\text { PVL group } \\
(\mathbf{N}=\mathbf{8})\end{array}$ & P-value \\
\hline SLLV, ml & 484.9 & 408.3 & \\
& $(396.5-568.1)$ & $(262.1-649.7)$ & 0.157 \\
Ratio of SLLV, \% & 47.1 & 40.0 & 0.122 \\
IntraoperativelCG-R15, \% & $(41.0-52.5)$ & $(23.0-57.6)$ & \\
& 13.4 & 17.0 & 0.081 \\
Laparoscopic surgery & $(12.0-15.8)$ & $(10.4-24.1)$ & NA \\
Operative time, minutes & 0 & 5 & $<0.001$ \\
Blood loss, ml & 349.5 & 164.6 & \\
& $(293-408)$ & $(92-229)$ & 0.022 \\
PHLF, $n$ (\%) & 433.3 & 185.0 & NA \\
Bile leak, $n$ (\%) & $(200-600)$ & $(20-500)$ & NA \\
Failure for Stage 2, n (\%) & $2(33.3 \%)$ & 0 & NA \\
\hline
\end{tabular}

ALPPS, associating liver partition and portal vein ligation for staged hepatectomy; PVL, portal vein ligation.

TABLE 5 | Comparison of staged-2 clinical characteristics of $\mathrm{SRH}$ between the ALPPS group and PVL group.

\begin{tabular}{|c|c|c|c|}
\hline Variables & $\begin{array}{l}\text { ALPPS } \\
(N=6)\end{array}$ & $\begin{array}{l}\text { PVL group } \\
(N=5)\end{array}$ & $P$-value \\
\hline TACE after stage 1 & 0 & 2 & NA \\
\hline Time to second stage, days & 14.6(12-22) & $44.2(21-83)$ & 0.016 \\
\hline Increased in volume per day, $\mathrm{ml}$ & $\begin{array}{l}12.7 \\
(5.2-14.9)\end{array}$ & $9.8(1.2-18.6)$ & 0.522 \\
\hline Ratio of gained standard volume per day, & $\%$ 1.2(0.5-1.5) & $1.0(0.1-1.8)$ & 0.536 \\
\hline Operative time of stage 2 , minutes & $\begin{array}{l}338.6(140- \\
515)\end{array}$ & $\begin{array}{l}255.2(185- \\
450)\end{array}$ & 0.274 \\
\hline Blood loss, ml & $\begin{array}{l}1016.7 \\
(300-2,500)\end{array}$ & $\begin{array}{l}940.0 \\
(350-1,500)\end{array}$ & 0.855 \\
\hline PHLF, $n(\%)$ & $1(16.7 \%)$ & $2(40 \%)$ & 0.545 \\
\hline \multicolumn{4}{|l|}{ Clavien-Dindo grade } \\
\hline Grade $1 / 2$ & $3 / 3$ & $3 / 2$ & 1.000 \\
\hline Bile leak, $n(\%)$ & $3(50 \%)$ & $3(60 \%)$ & 1.000 \\
\hline Hospital stay after surgery, days & 11(8-19) & $20(8-47)$ & 0.230 \\
\hline Recurrence within 1 year, $n(\%)$ & $3(50 \%)$ & $1(20 \%)$ & 0.545 \\
\hline
\end{tabular}

(43.4 days) to undergo the stage-2 $\mathrm{RH}$ when compared with the ALPPS group (14.0 days) ( $p=0.016$, Table 5). There were no significant differences in increased volume per day, increased standard volume per day, operative time, blood loss, fibrosis scoring, Clavien-Dindo scoring after the operation, postoperative hospital stay, and HCC recurrence within 1 year between the two groups (Table 5).

\section{Clinical Outcomes of the Two Patients With TACE After PVL}

Two patients underwent two sessions of TACE after PVL to slow down tumor progression for the slow increase in volumes 
of remnant left hemiliver. Finally, the increased volume and SLLV ratio in one patient increased from $262.1 \mathrm{~mL}(23.0 \%)$ to $450.1 \mathrm{ml}(39.5 \%)$ and another patient increased from $374.8 \mathrm{ml}$ (37.0\%) to $476.1 \mathrm{ml}$ (47.0\%; Figures 2A,C). These two patients underwent stage-2 $\mathrm{RH}$ although the tumor sizes had increased slightly from $13.4 \mathrm{~cm}$ to $14.3 \mathrm{~cm}$, and from $4.0 \mathrm{~cm}$ to $5.3 \mathrm{~cm}$, respectively (Figures $\mathbf{2 B}, \mathbf{D}$ ).

\section{DISCUSSION}

Right hepatectomy is a major hepatectomy. In general, the accepted remnant left hemiliver volume for safe liver resection ranges from $20-30 \%$ in patients without any underlying liver diseases $(4,20-22)$. However, in patients with chronic hepatitis/cirrhosis, the recommended critical minimum ratio of left hemiliver volume is $40 \%$ to support postoperative liver function (22). Thus, precise measurement of the left hemiliver volume before hepatectomy is helpful and necessary. Three-dimensional CT reconstruction provides a good method to calculate left hemiliver volume (10). However, accurate volumetrics do not necessarily represent an adequate liver function in the remnant liver because patients with chronic hepatitis/cirrhosis have impaired liver functions. Thus, the risk associated with PHLF in hepatectomy cannot solely rely on precise volume measurement of the remnant liver, but also on the adequate assessment of effective hepatic function. It is wellknown that ICG-R15 $(11,13)$ and technetium-99m (Tc-99m) mebrofenin (23), a medical radioactive isotope, were commonly used for accurate assessment of liver function and risk prediction of PHLF occurrence. A clinical study presented that Tc-99m mebrofenin blood clearance rate at $15 \mathrm{~min}$ was significantly correlated with the 15-min ICG clearance rate (24). ICG-R15 was known to mainly measure the global liver function, but Tc-99m mebrofenin scintigraphy could calculate not only the global liver function but also the effective FRL function (25), which is probably helpful to assess the left hemiliver function and to select qualified patients for RH. However, due to the unavailability of Tc-99m mebrofenin in our hospital, intraoperative ICG-R15 of the left liver was conducted to help operative decision-making in selecting patients for stage- 1 or stage- $2 \mathrm{RH}$.

The Institutional Review Board of our hospital only allows surgeons to carry out RH when the preoperative ICG-R15 test is $\leq 10 \%$ and the SLLV ratio is over $40 \%$ in patients with a background of chronic hepatitis, as this puts the patients to be at low risks of PHLF. However, the preoperative ICG-R15 tests assess the function of the whole liver, including parts of the liver which are going to be resected in $\mathrm{RH}$. It is therefore the design of this study to carry out intraoperative ICG-R15 in patients who are planned to undergo RH. In this study, there were two groups of patients who were planned to undergo $\mathrm{RH}$, namely, the intraoperative ICG test group and the comparison group. The data from the former group was collected prospectively whereas those from the latter group were collected retrospectively. When the two groups were compared, the former group had a significantly ( $p=0.001)$ lower ratio of SLLV\% $(44.3 \pm 8.8 \%)$ than the latter group $(55.0 \pm 9.6 \%)$, and the preoperative ICG-R15\% was significantly higher than the latter group [7.0(2.1-11.5)\% vs. 5.3(0.9-9.8)\%, $p=0.045]$. The hypothesis of this study is that the additional intraoperative ICG test of only the left hemiliver function would help to discriminate those who are at high risks of developing PHLF after RH (left-liver ICG-R15 > 10\%). Table 2 shows that intraoperative ICG-R15 helped to identify patients with adequate liver function to undergo RH. Only Grade A and B PHLF developed in those patients with incidences that were similar to those patients who were assessed preoperatively to have adequate volumes and functions to undergo $\mathrm{RH}$. This study also showed that intraoperative ICG-R15 is more accurate in assessing the function of the liver remnant as only the function of the left hemiliver was assessed intraoperatively, whereas all the non-tumorous parts of the whole liver were assessed by preoperative ICG-R15.

The preoperative ICG clearance test is commonly used and it has a high predictive value in assessing hepatic functional reserve, particularly in patients undergoing major hepatectomy (13). The future liver remnant plasma clearance rate of ICG (preoperative plasma clearance rate of indocyanine green $\times \%$ FRL) has been found useful in predicting post hepatectomy liver function $(26,27)$. In this study, the ICG-R15 clearance test was conducted after blocking the right hepatic blood flow to help decision-making for stage- 1 or stage- $2 \mathrm{RH}$ in 20 patients who preoperatively planned to undergo $\mathrm{RH}$. Based on a cut-off value of intraoperative ICG-R15 of $\leq 10 \%$, six patients underwent stage- $1 \mathrm{RH}$ and 14 were planned to undergo stage- $2 \mathrm{RH}$. Finally, 17 patients underwent $\mathrm{RH}$, including six for stage- $1 \mathrm{RH}$ and 11 for stage-2 RH. Although the degrees of fibrosis were more severe in the intraoperative ICG retention test group, clinical outcomes including incidences of PHLF, Clavien-Dindo classification of complications, hospital stay, and HCC recurrence within 1 year after stage- $1 \mathrm{RH}$ and stage-2 $\mathrm{RH}$ were similar. Using the cutoff value of $\leq 10 \%$ for intraoperative ICG-R15, 11 patients underwent stage-2 RH whereas six underwent stage-1 RH. There were no significant differences in SLLV and SLLV ratio between these two groups. However, the preoperative ICG-R15 and intraoperative ICG-R15 in those patients who underwent stage-2 RH were significantly different from those patients who underwent stage- $1 \mathrm{RH}$ ( $p=0.01$ and $P<0.001$, respectively). All the 11 patients underwent stage- $2 \mathrm{RH}$ after adequate increase in volumes of remnant left hemiliver. The incidence of PHLF was lower than stage-1 RH. These results showed that "both volume and function" are important, and intraoperative ICG retention test is helpful in intraoperative decision-making for either stage1 or stage- $2 \mathrm{RH}$ for patients who are considered to undergo $\mathrm{RH}$ to avoid severe PHLF and posthepatectomy complications. Our data also revealed that PHLF after RH was closely related to the necroinflammatory scoring in patients with intraoperative ICG-R15 $\leq 10 \%$ of left hemiliver and SLLV $\geq 40 \%(r=0.404$, $p=0.045)$. The liver fibrosis score was correlated with the necroinflammatory score $(r=0.527, p=0.007)$.

Our results demonstrated that the operative time and blood loss during stage-1 ALPPS were significantly more than in stage$1 \operatorname{PVL}(p<0.001$ and $p=0.022$, respectively). Furthermore, two patients $(33.3 \%)$ suffered from PHLF and one patient $(16.7 \%)$ developed bile leakage after stage-1 ALPPS. There was also no 


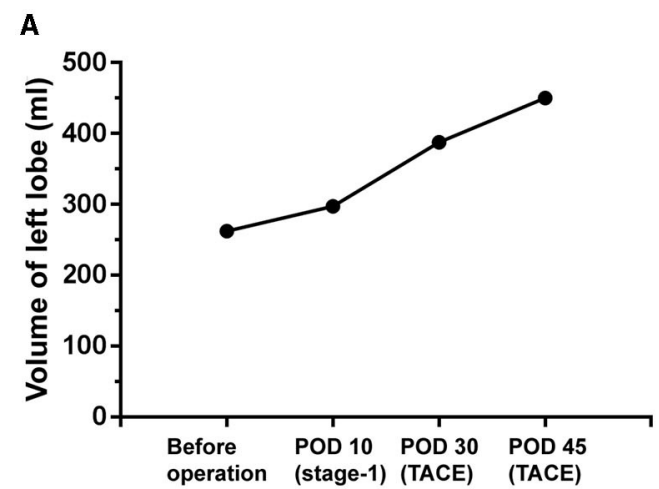

Case 1

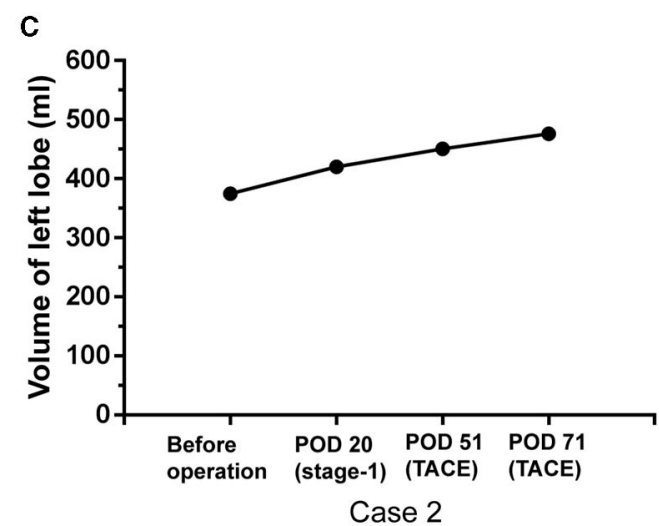

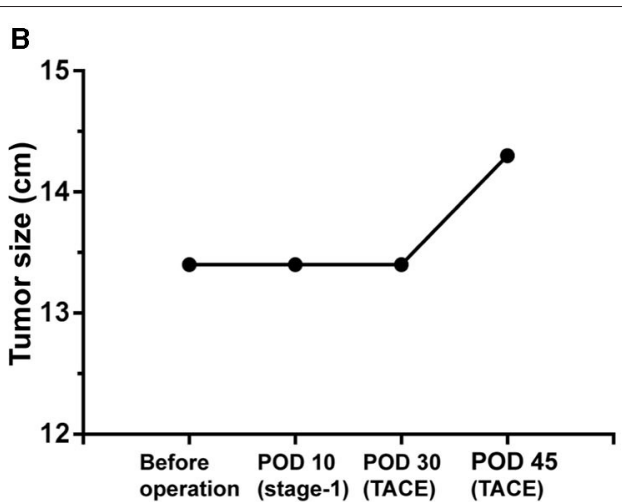

Case 1

D

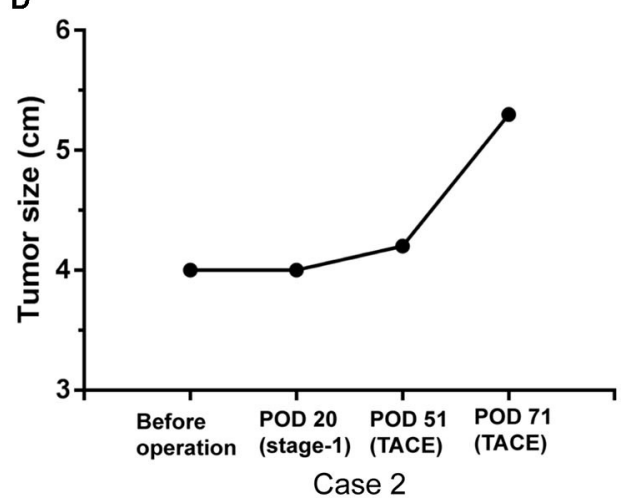

FIGURE 2 | Volume increase and tumor progression in two patients with TACE after PVL. Volume increase of left lobe (A) and tumor size change (B) after PVL and TACE in the first case, volume increase of left lobe (C) and tumor size change (D) after PVL and TACE in the second case. POD: postoperative day.

complication in the stage-1 PVL, indicating that PVL is safer. However, patients in the ALPPS group spent significantly shorter waiting time and more patients were able to proceed to stage- 2 $\mathrm{RH}$ compared with the PVL group, suggesting that ALPPS was a better choice for completion of the stage-2 RH than PVL. The price to pay for stage-1 ALPPS was a higher probability of PHLF and bile leakage. These results were consistent with the previously reported study that ALPPS had a significantly higher rate of complete liver resection in patients with initially unresectable liver tumors with inadequate volumes of the remnant liver when compared with PVE/PVL, but at the cost of higher complication and mortality rates (28).

In this small-sample-size study, although patients in the PVL group had to wait for a significantly longer time (43.4 days) for stage-2 RH compared with the ALPPS group (14.0 days), PVL had the advantages of less operative time and less blood loss. Three patients failed to reach to stage-2 $\mathrm{RH}$ due to tumor progression on days- $4,15,21$, respectively after stage-1 PVL. There was a significantly lower tumor recurrence rate within 1 year in the stage-1 PVL group $(20 \%, 1 / 5)$ when compared with the stage-1 ALPPS group (50\%, 3/6). However, whether this stage-1 PVL can be used as a "wait and see" strategy to discriminate HCC patients with more biologically favorable HCC to undergo stage-2 $\mathrm{RH}$ requires large-sample-sized studies to confirm.
A previously reported study showed that rescue TACE was helpful to increase the waiting time for the future liver remnant to grow adequately in size for stage- 2 hepatectomy after laparoscopic-associated liver tourniquet and portal ligation (29). In our study, two patients underwent TACE after stage-1 PVL, and the procedure was effective in limiting tumor progression to wait for the remnant left hemiliver to increase adequately in volume. Finally, these two patients successfully completed stage-2 RH. Regrettably, one had a tumor recurrence within a short period of time ( 7 months) after stage-2 RH.

In conclusion, our results suggested that intraoperative ICG$\mathrm{R} 15$ is helpful in the decision-making of stage-1 or stage$2 \mathrm{RH}$ for HCC patients. An intraoperative ICG-R15 $\leq 10 \%$ of left hemiliver was proposed to be the good cut-off value for safe RH. However, the present study has the following limitations. First, the sample size of the study is small, future large-sample-size studies are needed to verify the findings of this study. Second, although part of the study was prospective (the intraoperative ICG-R15 study group), part of it was retrospective (the comparison group). There are potential inherent biases in such a type of study. Third, all the patients in this study were patients with HBV-related HCC. The results of this study may not be extrapolated to patients with other etiologies of HCC. Finally, this study was conducted on patients with HCC who were 
planning to undergo $\mathrm{RH}$. The results may not be applicable to other types of liver resection.

\section{DATA AVAILABILITY STATEMENT}

The original contributions presented in the study are included in the article/supplementary material, further inquiries can be directed to the corresponding author/s.

\section{ETHICS STATEMENT}

The studies involving human participants were reviewed and approved by the Ethics Committee of the First Affiliated Hospital of Guangxi Medical University. The patients/participants provided their written informed consent to participate in this study.

\section{AUTHOR CONTRIBUTIONS}

TP designed the study and revised the manuscript. TY analyzed the data and wrote the manuscript. XY codesigned the study and performed an intraoperative indocyanine green retention test. WL revised the manuscript and language editing. ZW, GZ, HS, and BX provided study patients and carried

\section{REFERENCES}

1. Bruix J, Sherman M. Management of hepatocellular carcinoma: an update. Hepatology. (2011) 53:1020-2. doi: 10.1002/hep.24199

2. Clavien PA, Petrowsky H, DeOliveira ML, Graf R. Strategies for safer liver surgery and partial liver transplantation. N Engl J Med. (2007) 356:154559. doi: 10.1056/NEJMra065156

3. Farges O, Malassagne B, Flejou JF, Balzan S, Sauvanet A, Belghiti J. Risk of major liver resection in patients with underlying chronic liver disease: a reappraisal. Ann Surg. (1999) 229:2105. doi: 10.1097/00000658-199902000-00008

4. Schindl MJ, Redhead DN, Fearon KC, Garden OJ, Wigmore SJ. The value of residual liver volume as a predictor of hepatic dysfunction and infection after major liver resection. Gut. (2005) 54:289-96. doi: 10.1136/gut.2004.046524

5. Levi Sandri GB, Colasanti M, Vennarecci G, Santoro R, Lepiane P, Mascianà $\mathrm{G}$, et al. A 15-year experience of two hundred and twenty five consecutive right hepatectomies. Dig Liver Dis. (2017) 49:50-6. doi: 10.1016/j.dld.2016.09.014

6. Makuuchi M, Thai BL, Takayasu K, Takayama T, Kosuge T, Gunvén P, et al. Preoperative portal embolization to increase safety of major hepatectomy for hilar bile duct carcinoma: a preliminary report. Surgery. (1990) 107:521-7.

7. Aussilhou B, Lesurtel M, Sauvanet A, Farges O, Dokmak S, Goasguen N, et al. Right portal vein ligation is as efficient as portal vein embolization to induce hypertrophy of the left liver remnant. J Gastrointest Surg. (2008) 12:297-303. doi: 10.1007/s11605-007-0410-x

8. de Santibañes E, Clavien PA. Playing Play-Doh to prevent postoperative liver failure: the "ALPPS" approach. Ann Surg. (2012) 255:415-7. doi: 10.1097/SLA.0b013e318248577d

9. Marescaux J, Clément JM, Tassetti V, Koehl C, Cotin S, Russier Y, et al. Virtual reality applied to hepatic surgery simulation: the next revolution. Ann Surg. (1998) 228:627-34. doi: 10.1097/00000658-199811000-00001

10. Wigmore SJ, Redhead DN, Yan XJ, Casey J, Madhavan K, Dejong CH, et al. Virtual hepatic resection using three-dimensional reconstruction of helical computed tomography angioportograms. Ann Surg. (2001) 233:2216. doi: 10.1097/00000658-200102000-00011

11. Zipprich A, Kuss O, Rogowski S, Kleber G, Lotterer E, Seufferlein T, et al. Incorporating indocyanin green clearance into the Model for End Stage out the intraoperative indocyanine green retention test. $\mathrm{CH}$, $\mathrm{KH}$, and WQ performed the liver volume measurement and checked the data. XL, CY, ZL, and XW performed preoperative indocyanine green retention test and clinical data collection. ZL completed pathological diagnosis and MVI assessment. All authors contributed to the article and approved the submitted version.

\section{FUNDING}

This work was supported in part by the National Nature Science Foundation of China (No. 81560535, 81072321, 30760243, 30460143, and 30560133), 2009 Program for New Century Excellent Talents in University (NCET), Guangxi Nature Sciences Foundation (No. GuiKeGong 1104003A-7), and Guangxi Health Ministry Medicine Grant (Key-Scientific Research-Grant Z201018).

\section{ACKNOWLEDGMENTS}

The authors thank Min-Hao Peng, Kai-Yin Xiao, Xi-Gang Chen, Bin Chen, and Li-Ming Shang (Department of Hepatobiliary Surgery, the First Affiliated Hospital of Guangxi Medical University) for providing patients for this study.
Liver Disease (MELD-ICG) improves prognostic accuracy in intermediate to advanced cirrhosis. Gut. (2010) 59:963-8. doi: 10.1136/gut.2010.208595

12. de Liguori Carino N, O’Reilly DA, Dajani K, Ghaneh P, Poston GJ, Wu AV. Perioperative use of the LiMON method of indocyanine green elimination measurement for the prediction and early detection of post-hepatectomy liver failure. Eur J Surg Oncol. (2009) 35:957-62. doi: 10.1016/j.ejso.2009.02.003

13. Lam CM, Fan ST, Lo CM, Wong J. Major hepatectomy for hepatocellular carcinoma in patients with an unsatisfactory indocyanine green clearance test. Br J Surg. (1999) 86:1012-7. doi: 10.1046/j.1365-2168.1999.01204.x

14. Zhou J, Sun HC, Wang Z, Cong WM, Wang JH, Zeng MS, et al. Guidelines for diagnosis and treatment of primary liver cancer in China (2017 Edition). Liver Cancer. (2018) 7:235-60. doi: 10.1159/0004 88035

15. Forner A, Reig ME, de Lope CR, Bruix J. Current strategy for staging and treatment: the BCLC update and future prospects. Semin Liver Dis. (2010) 30:61-74. doi: 10.1055/s-0030-12 47133

16. Ishak K, Baptista A, Bianchi L, Callea F, De Groote J, Gudat F, et al. Histological grading and staging of chronic hepatitis. J Hepatol. (1995) 22:696-9. doi: 10.1016/0168-8278(95)80226-6

17. Rahbari NN, Garden OJ, Padbury R, Brooke-Smith M, Crawford M, Adam $\mathrm{R}$, et al. Posthepatectomy liver failure: a definition and grading by the International Study Group of Liver Surgery (ISGLS). Surgery. (2011) 149:71324. doi: 10.1016/.j.surg.2010.10.001

18. Dindo D, Demartines N, Clavien PA. Classification of surgical complications: a new proposal with evaluation in a cohort of 6336 patients and results of a survey. Ann Surg. (2004) 240:205-13. doi: 10.1097/01.sla.0000133083.54 934.ae

19. Fu-Gui L, Lu-Nan Y, Bo L, Yong Z, Tian-Fu W, Ming-Qing X, et al. Estimation of standard liver volume in Chinese adult living donors. Transplant Proc. (2009) 41:4052-6. doi: 10.1016/j.transproceed.2009. 08.079

20. Kishi Y, Abdalla EK, Chun YS, Zorzi D, Madoff DC, Wallace MJ, et al. Three hundred and one consecutive extended right hepatectomies: evaluation of outcome based on systematic liver volumetry. Ann Surg. (2009) 250:5408. doi: 10.1097/SLA.0b013e3181b674df 
21. Facciuto M, Contreras-Saldivar A, Singh MK, Rocca JP, Taouli B, Oyfe I, et al. Right hepatectomy for living donation: role of remnant liver volume in predicting hepatic dysfunction and complications. Surgery. (2013) 153:61926. doi: 10.1016/j.surg.2012.11.020

22. Guglielmi A, Ruzzenente A, Conci S, Valdegamberi A, Iacono C. How much remnant is enough in liver resection? Dig Surg. (2012) 29:617. doi: 10.1159/000335713

23. Dasari BVM, Wilson M, Pufal K, Kadam P, Hodson J, Roberts KJ, et al. Variations between the anatomical and functional distribution, based on $99(\mathrm{~m})$ technetium -mebrofinate SPECT-CT scan, in patients at risk of post hepatectomy liver failure. HPB. (2021) S1365-182X(21)001143. doi: 10.1016/j.hpb.2021.04.014

24. Erdogan D, Heijnen BH, Bennink RJ, Kok M, Dinant S, Straatsburg IH, et al. Preoperative assessment of liver function: a comparison of $99 \mathrm{mTc}$ Mebrofenin scintigraphy with indocyanine green clearance test. Liver Int. (2004) 24:117-23. doi: 10.1111/j.1478-3231.2004.00901.x

25. de Graaf W, van Lienden KP, van Gulik TM, Bennink RJ. (99m)Tc-mebrofenin hepatobiliary scintigraphy with SPECT for the assessment of hepatic function and liver functional volume before partial hepatectomy. J Nucl Med. (2010) 51:229-36. doi: 10.2967/jnumed.109.069724

26. Yokoyama Y, Nishio H, Ebata T, Igami T, Sugawara G, Nagino M. Value of indocyanine green clearance of the future liver remnant in predicting outcome after resection for biliary cancer. Br J Surg. (2010) 97:12608. doi: 10.1002/bjs.7084

27. Uchida Y, Furuyama H, Yasukawa D, Nishino H, Ando Y, Hata $\mathrm{T}$, et al. Hepatectomy based on future liver remnant plasma clearance rate of indocyanine green. HPB Surg. (2016) 2016:7637838. doi: $10.1155 / 2016 / 7637838$
28. Schadde E, Ardiles V, Slankamenac K, Tschuor C, Sergeant G, Amacker $\mathrm{N}$, et al. ALPPS offers a better chance of complete resection in patients with primarily unresectable liver tumors compared with conventional-staged hepatectomies: results of a multicenter analysis. World J Surg. (2014) 38:15109. doi: 10.1007/s00268-014-2513-3

29. Xu J, Lu X, Zhang Y, Yang H, Yu X. Laparoscopic associating liver tourniquet and portal ligation combined rescue transhepatic arterial embolization for staged hepatectomy: a case report. Medicine. (2017) 96:e8378. doi: 10.1097/MD.0000000000008378

Conflict of Interest: The authors declare that the research was conducted in the absence of any commercial or financial relationships that could be construed as a potential conflict of interest.

Publisher's Note: All claims expressed in this article are solely those of the authors and do not necessarily represent those of their affiliated organizations, or those of the publisher, the editors and the reviewers. Any product that may be evaluated in this article, or claim that may be made by its manufacturer, is not guaranteed or endorsed by the publisher.

Copyright (C) 2021 Yu, Ye, Wen, Zhu, Su, Han, Huang, Qin, Liao, Yang, Liu, Wang, $X u, S u, L v, L a u$ and Peng. This is an open-access article distributed under the terms of the Creative Commons Attribution License (CC BY). The use, distribution or reproduction in other forums is permitted, provided the original author(s) and the copyright owner(s) are credited and that the original publication in this journal is cited, in accordance with accepted academic practice. No use, distribution or reproduction is permitted which does not comply with these terms. 